\title{
Unintentional Injuries among School-Aged Children in Palestine: Findings from the National Study of Palestinian Schoolchildren (HBSC-WBG2006)
}

\author{
Christine Jildeh, ${ }^{1}$ Ziad Abdeen, ${ }^{2,3}$ Haleama Al Sabbah, ${ }^{4,5}$ and Anastas Philalithis ${ }^{1}$ \\ ${ }^{1}$ Department of Social Medicine, School of Medicine, University of Crete, P.O. Box 2208, 71003 Iraklion, Crete, Greece \\ ${ }^{2}$ The Al-Quds Nutrition and Health Research Center, Al-Quds University, P.O. Box 20760, Jerusalem, Palestine \\ ${ }^{3}$ Faculty of Medicine, Al-Quds University, P.O. Box 51000, Jerusalem, Palestine \\ ${ }^{4}$ Faculty of Medicine, An-Najah National University, P.O. Box 7, Nablus, West Bank, Palestine \\ ${ }^{5}$ Jean Mayer USDA Human Nutrition Research Centre, Tufts University, 711 Washington street, Boston, MA 02111, USA \\ Correspondence should be addressed to Ziad Abdeen; zabdeen@planet.edu
}

Received 26 September 2012; Accepted 19 February 2013

Academic Editor: Sally Guttmacher

Copyright (C) 2013 Christine Jildeh et al. This is an open access article distributed under the Creative Commons Attribution License, which permits unrestricted use, distribution, and reproduction in any medium, provided the original work is properly cited.

Purpose. This study describes the nonfatal injuries among adolescents in Palestine. Methods. The 2006 Palestinian Health Behaviour in School-aged Children (HBSC) is a cross-sectional survey. Students of grades 6, 8, 10, and 12 completed a modified version of the international HBSC questionnaire, resulting in 15,963 students (47.3\% boys and $52.7 \%$ girls) included in this study (56.9\% from the West Bank and 43.1\% from Gaza). Results. Of the total 15,963 adolescents, $47.6 \%$ were injured, with boys (53.5\%) being statistically higher than girls $(42.1 \%)(P<0.001)$. The prevalence of those injured more than once decreased by age and was also found significantly higher in boys than in girls $(27.3 \%$ and $17.9 \%$, resp. $)(P<0.001)$. Children living in low FAS families showed significantly lower ratios of injuries than those living in moderate and high FAS families $(P<0.001)$. Injuries while biking were significantly higher among boys $(46.3 \%)$ than girls $(41.7 \%)(P<0.001)$, and injuries while walking/running were more prevalent among girls $(32.5 \%)$ than boys $(28.0 \%)(P<0.001)$. Conclusion. Despite these considerably high rates, injury remains relatively underappreciated. Results of this study are useful to develop a national injury prevention program aimed at enhancing the safety of Palestinian adolescents.

\section{Introduction}

Injuries are the world's leading cause of morbidity and mortality among children and adolescents [1]. According to the World Health Organization report of 2008, injury is a leading cause of the global burden of disease; injuries are responsible for more than 950,000 deaths every year among those under 19 years of age, from which at least 60 percent are attributed to unintentional causes. However, the differences such as the extent, pattern, distribution, risk, and determinants between the intentional and unintentional home, school and roadrelated childhood, and young adulthood injury hazards are not well understood [2].

Many studies had focused on the typology of injuries and their socio-economic status correlates $[3,4]$ particular types of injuries, such as sport injuries [5], the association between family stress and injuries [6], risk behaviors and injuries [7], violence and injuries $[4,8]$, and other psychosocial determinants $[4,9,10]$. Despite the enormous public health burden imposed by child and adolescent injury, several studies had shown that $71-95 \%$ of injuries could be prevented by simple and reasonable means $[11,12]$. In developed countries such as Australia, Sweden, Britain, and Canada, injury rates has been reduced by up to $50 \%$ over the past three decades using multisectored approaches to prevention [13]. In developing countries, studies have been largely limited to hospital-based information which provides information on severe injuries $[14,15]$.

A population-based survey of adults $18-65$ years residing in Aleppo, Syria, conducted in 2004 founds that falls and traffic injuries have caused the highest morbidity ratios 
among the injured, while burns, although not frequently reported, were associated with the severity of injury in the majority of cases [16]. A recent report in 2008 about the incidence of home accidents in Palestinian and Israeli communities stated that falls were the most common cause of injury reported by parents surveyed (35\% in the Palestinian community and $31 \%$ in the Bedouin community); the majority of these accidents occurred among boys ( $60 \%$ in the Bedouin community and $70 \%$ in the Palestinian community) with the yard, living room, and kitchen as places with greatest number of accidents [17]. Another main site of accidents for the Palestinian population was the stairs-a site not relevant in the Bedouin homes. In the Bedouin community, burns represented an equal proportion of injuries to falls, significantly higher than the proportion found in the Palestinian survey (31\% versus $11 \%$ ) or in the international literature. Analysis of the circumstances of these accidents reveals that most were related to the use of an open fire for cooking and heating, which are common to the Bedouin lifestyle [17].

The Palestinian Ministry of Health (PMOH) reported that in 2004 accidents were the leading cause of death for children aged 1-4 and 5-19 years with a proportion of $24.2 \%$, and $29.2 \%$, respectively of the total deaths [18]. And although children constitute $52.6 \%$ of the Palestinian total population [19], there is little literature on unintentional injuries in Palestine. Therefore, the Palestinian Health Behavior in School-aged Children (PHBSC) survey offers for the first time the opportunity to study the Palestinian adolescents' unintentional injuries in grades $6,8,10$, and 12 . The intent of this analysis was to estimate the prevalence rate of injuries, severity of injuries, settings in which injuries occur, and the activity context of injury events occurring to Palestinian adolescents as well as to compare the results of this study with studies from other countries which used similar type of research and target group to be used as a model to develop future injury surveillance methods to track injuries and their risk factors. This study is essential for understanding the extent of the injuries problem to inform both public policy and prevention strategies.

\section{Methods}

2.1. Target Population and Sample. The estimated population of Palestine in 2006 was 3,761,646 people (West Bank = 2,345,107 and Gaza Strip =1,416,539) [20]; the total numbers of youth aged 10-14 and 15-19 were 514,997 and 429,276, respectively. The schooling system in Palestine is comprised of two stages: a basic stage that involves children aged 6 to 16 years and a secondary stage of children aged 17 to 18 years. In 2006, there were 2,277 schools in Palestine (1726 government-run, 279 United Nations Relief and Works Agency (UNRWA), which provides education, among other services, to Palestinian refugees living in the Gaza Strip and the West Bank, and 272 private schools) with a total number of 31,019 classes (22,110 government, 6107 UNRWA, 2802 private) [21]. The total number of children enrolled in basic education during the scholastic year 2005/2006 was 944,713 , while 122,776 students were enrolled in the secondary education [21].

Selected students were chosen using a stratified, twostage cluster sampling. The sample was stratified according to region (West Bank and Gaza Strip), school types (government, private, and UNRWA), and school grades (6th, 8th, 10 th, and 12th). Assuming an average class size of 35 students, the aim was to recruit 4,000 students at each grade level, from both regions, to produce a sample size of around 16,000 students. A two-stage cluster sampling technique was used with the school as the primary sampling unit. In the first stage, 405 schools were selected randomly with probability proportional to their size (size defined as number of classes in the school). The list of the schools and number of classes in each school, for the academic year 2005/2006, were obtained from the Ministry of Education. All of the randomly selected schools $(n=405)$ agreed to participate. The response rate was $97.3 \%$ among 16,400 students who participated on data collection, leaving a sample size of 15,963 . In the second stage, one class was selected at random from each school. Each of these classes was defined as a cluster, where all the students in the selected class were eligible for inclusion. The aim was to survey one class per school. However, in schools where boys and girls were studying in separate classes, two classes were selected, one for boys and another for girls. This resulted in a total of 481 classes being selected overall.

2.2. Data Collection and Questionnaire. The questionnaire was developed using the WHO international HBSC questionnaire (2001-2002) including all mandatory HBSC questions [22]. The questionnaire was double cross translated to Arabic; it was tested in two independent preliminary studies using in-class administration as well as focus-group discussions to demonstrate reliability and validity prior to the administration of the national representative full survey.

In addition to the major variables addressed in the survey (demographics, general health, well-being, family and peer relationships, school environment, exercise and leisure-time activities, diet, smoking, exposure to political violence, and mental health) [23-25], the questionnaire contained additional optional packages; half of the optional components of the questionnaire (Form A) contained optional questions on violence, injuries, and social inequalities, and the other half of the questionnaire (Form B) contained optional questions on physical activity, eating, dieting, and mental and physical health. Equal numbers of Form A and Form B were randomly distributed in each school class. However, the injury filter items were asked to all sampled students. The survey was conducted anonymously in the West Bank and Gaza through self-completed questionnaires between April-May of 2006.

In each school, the student advisor distributed the questionnaires in the classroom and was instructed to respond to the students' enquiries about the procedure. School children completed the questionnaires independently during one school period, lasting 45 minutes. Data collection was conducted by Al-Quds Nutrition and Health Research Institute (ANAHRI) in Al-Quds University. 


\subsection{Main Measures}

2.3.1. Injury. Students were asked to report injury events that required medical attention from a doctor or a nurse in the past 12 months [26]. Response options were "I was never injured in the past 12 months," "once," "twice," "three times" and "four times or more". Students who reported one or more injuries were defined as being injured. Respondents who reported at least one injury were requested to answer a series of questions related to the circumstances and consequences of the injury event. Respondents who reported two or more injuries were asked to select the "single most serious injury during the past 12 months" and were requested to answer the following questions referring to that "most serious injury". The most serious injury was defined as the injury that took the most time to get better [10, 27-29].

The information obtained referred to the timing of the injury (month and year), setting where the injury occurred (e.g., home, school, street), the activity context (e.g., biking, sports, fighting), and consequence (e.g., loss of activity days or school days, hospitalization, etc.).

2.3.2. Family Affluence Scale (FAS). Family Affluence Scale (FAS) was developed by the WHO Health Behaviour in School-Aged Children Study. This scale deduced the levels of material wealth by calculating points to "owning a car," "owning a computer," "sharing a bedroom," and "travelling while on holiday." A sum score was calculated for each student and categorized as (i) low FAS (score $=0-2$ ); (ii) medium FAS (score $=3-5)$; (iii) high FAS (score $=6-9)$ [30].

2.3.3. Parents' Educational Level. This is based on adolescents self-reports and is divided into four levels: (1) did not graduate from high school; (2) graduated from high school; (3) continued studies after high school but not in the university; (4) studied in university or graduated from university. Responses are categorized into (A) low education-a combination of $1+$ 2 and $(\mathrm{B})$ high education $-\mathrm{a}$ combination of $3+4$.

2.3.4. Parents' Occupation. A more direct measure of the family economic situation was derived from a question reporting the current occupation status of the parents. This is based on adolescents self-reports and responses were 1yes; 2-no; 3-do not know; 4-do not have/do not see the father/mother. In the analysis, do not know or do not have/do not see the father/mother were combined in one category.

2.3.5. Activity During Injuries. Participants were asked to choose their activity when the accident occurred. Response options included "biking/cycling, sports activity/recreational activity, walking/running (not for a sports team or exercise), fighting, paid or unpaid work, and other." Location of injuries was asked, and the response options included "home or yard, school, including school grounds, sports facilities, or fields, street, and road."

2.4. Ethical Considerations. The 2006 Palestinian HBSC survey was approved by Al-Quds University Ethical Committee and the Research Ethics Board of the Palestinian Ministry of Education.

2.5. Statistical Analysis. Percentages for injuries have been calculated on the basis of sociodemographic characteristics (grade, gender, region, school ownership, and FAS). Chisquare test was used to compare differences in injuries among adolescents by sociodemographic characteristics. Analysis was performed using SPSS Version 20. A significance level of $P<0.05$ was used.

\section{Results}

The total number of students included in this study is 15,963 , from which $52.7 \%$ are girls and $47.3 \%$ are boys. More than half the participants resided in the West Bank (56.9\%). The majority of the students' fathers and mothers had low education level (75.6\% and $65.5 \%$, resp.), and $71.8 \%$ of the fathers were working, while $71.5 \%$ of mothers were housewives. Based on the Family Affluence Scale (FAS), 66.6\% of the students' families have low economic situation and $79.5 \%$ of the adolescents attend government schools (Table 1).

Table 2 presents the percent of students reporting one or more medically attended injury during the past 12 month by grade, gender, region, school ownership, and FAS. Of the total $(15,963)$ participants, $47.6 \%$ were injured $(53.5 \%$ boys versus $42.1 \%$ girls). About one quarter (25.2\%) of the participants reported one injury and with boys higher than girls $(26.2 \%$ and $24.2 \%$, resp.) $(P<0.001)$. Whereas, $22.4 \%$ reported two or more medically attended injuries during the past 12 months. The prevalence of those injured more than once decreased by age and was found significantly higher in boys than in girls $(27.3 \%$ and $17.9 \%$, resp. $)(P<0.001)$. Adolescents attending UNRWA schools showed significantly higher ratios of injuries in both single and multiple injures than other schools. Adolescents living in families with low FAS reported significantly lower ratios of injuries than those living with moderate and high FAS families $(P<0.001)$.

Loss of activity days due to the injury was used as an indicator of more severe injuries. About half of all reported injuries $(42.4 \%)$ were severe enough to cause loss of at least one day of school or other activities. This proportion significantly decreased by grade $(P<0.001)$ and was significantly higher among boys than girls (47.2\% and $37.0 \%$ resp.) $(P<0.001)$.

The most frequent activity in which injuries occurred was biking (44.2\%) and among boys (46.3\%) more than among girls (41.7\%). Injuries while walking/running were more prevalent among girl (32.5\%) than among boys (28.0\%). About one out of every five injury events occurred during fighting.

Home injuries has the highest ratio (78.5\%) followed by school (9.0\%) and streets (4.2\%) (Figure 1). There was no significant difference between the injury setting (home, school, sport facility, and street) and the sociodemographic characteristics (age, gender, region, school ownership, and FAS), while there was significant difference between organized activity and injury setting with sport facilities being the highest $47.0 \%$ (Figure 2). 
TABLE 1: Characteristics of participants $(n=15963)$.

\begin{tabular}{|c|c|c|}
\hline Characteristics & $n$ & $\%$ \\
\hline \multicolumn{3}{|l|}{ Grade } \\
\hline 6th & 4263 & 26.7 \\
\hline 8 th & 4124 & 25.8 \\
\hline 10th & 4113 & 25.8 \\
\hline 12 th & 3463 & 21.7 \\
\hline \multicolumn{3}{|l|}{ Gender } \\
\hline Boys & 7551 & 47.3 \\
\hline Girls & 8412 & 52.7 \\
\hline \multicolumn{3}{|l|}{ Region } \\
\hline West Bank & 9077 & 56.9 \\
\hline Gaza Strip & 6886 & 43.1 \\
\hline \multicolumn{3}{|l|}{ School ownership } \\
\hline Private & 1243 & 7.8 \\
\hline Government & 12688 & 79.5 \\
\hline UNRWA* & 2032 & 12.7 \\
\hline \multicolumn{3}{|l|}{ Family Affluence Scale (FAS) } \\
\hline FAS 1 (low) & 9494 & 66.6 \\
\hline FAS 2 (moderate) & 3259 & 22.9 \\
\hline FAS 3 (high) & 1502 & 10.5 \\
\hline \multicolumn{3}{|l|}{ Father's education } \\
\hline Low education & 10833 & 75.6 \\
\hline High education & 3488 & 24.4 \\
\hline \multicolumn{3}{|l|}{ Mother's education } \\
\hline Low education & 9466 & 65.5 \\
\hline High education & 4980 & 34.5 \\
\hline \multicolumn{3}{|l|}{ Father's occupation } \\
\hline Yes & 10398 & 71.8 \\
\hline No & 3006 & 20.8 \\
\hline $\begin{array}{l}\text { Do not know or do not have/do not see the } \\
\text { father }\end{array}$ & 1075 & 7.4 \\
\hline \multicolumn{3}{|l|}{ Mother's occupation } \\
\hline Yes & 3054 & 21.2 \\
\hline No & 10280 & 71.5 \\
\hline $\begin{array}{l}\text { Do not know or do not have/do not see the } \\
\text { mother }\end{array}$ & 1044 & 7.2 \\
\hline
\end{tabular}

${ }^{*}$ UNRWA: United Nation Relief Working Agency.

\section{Discussion}

The overall prevalence of Palestinian adolescents who were injured at least once during the past 12 months from conducting the survey was $47.6 \%$ of the total, and injuries among boys (53.5\%) were higher than among girls (42.1\%). These results are consistent with results from European countries, USA, and Israel that boys have more injuries than girls $[4,9,10$, $16,31-33]$.

Although there was variation between countries, injuries between Palestinian adolescents were less than Spain (63.9\% boys and $50.7 \%$ girls), Germany (58.1\% boys and $50.3 \%$ girls), Austria (58.0\% boys and $47.9 \%$ girls), USA (56.9\% boys and

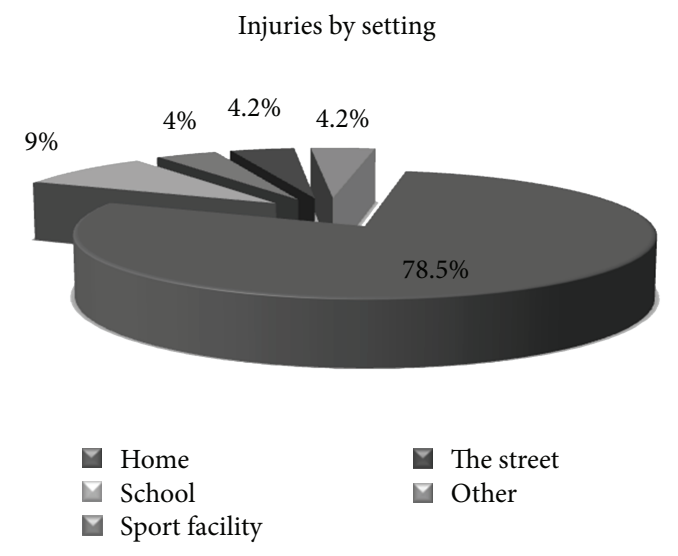

FIgURE 1: This graph shows the prevalence of injury by setting.

$$
\text { Injuries during organized activity by injury setting }
$$

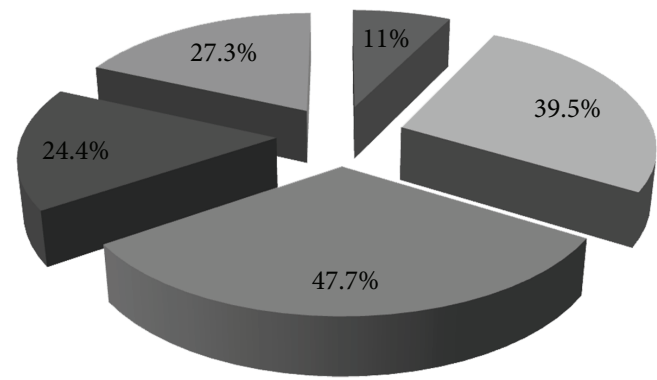

$$
\begin{aligned}
& \text { Home } \\
& \text { School } \\
& \text { Sport facility }
\end{aligned}
$$

FIGURE 2: This graph shows the prevalence of injury according to injury setting as well as the percentage of injuries during organized activity in each category.

$47.6 \%$ girls), and Israel ( $62.1 \%$ boys and $46.3 \%$ girls) [ 4 ]. While the results from this study were closer to results from Slovenia (54.3\% boys and $40.3 \%$ girls) and Switzerland (54.0\% boys and $43.1 \%$ girls). Furthermore, this showed higher prevalence of injuries than Poland (33.4\% boys and $23.3 \%$ girls), Ukraine (37.2\% boys and $24.6 \%$ girls), and Greenland (36.4\% boys and $25.5 \%$ girls) [4].

The operational definition of an injury in this study is an injury that was treated by a doctor or a nurse. Therefore, the relatively lower-than-expected injury rate shown for Palestinian students compared to some other European countries, Israel, and USA can be explained by one or more of the following reasons: (1) lower injury rates, (2) less access to medical care, and (3) less utilization of medical care. Consequently, unattended Palestinian adolescents' injuries might be underreported.

This study found that adolescents living in high FAS families reported high prevalence of injures (51.9\%) while those living in low FAS families reported low injuries (45.1\%), which is consistent with the results from the cross-national 


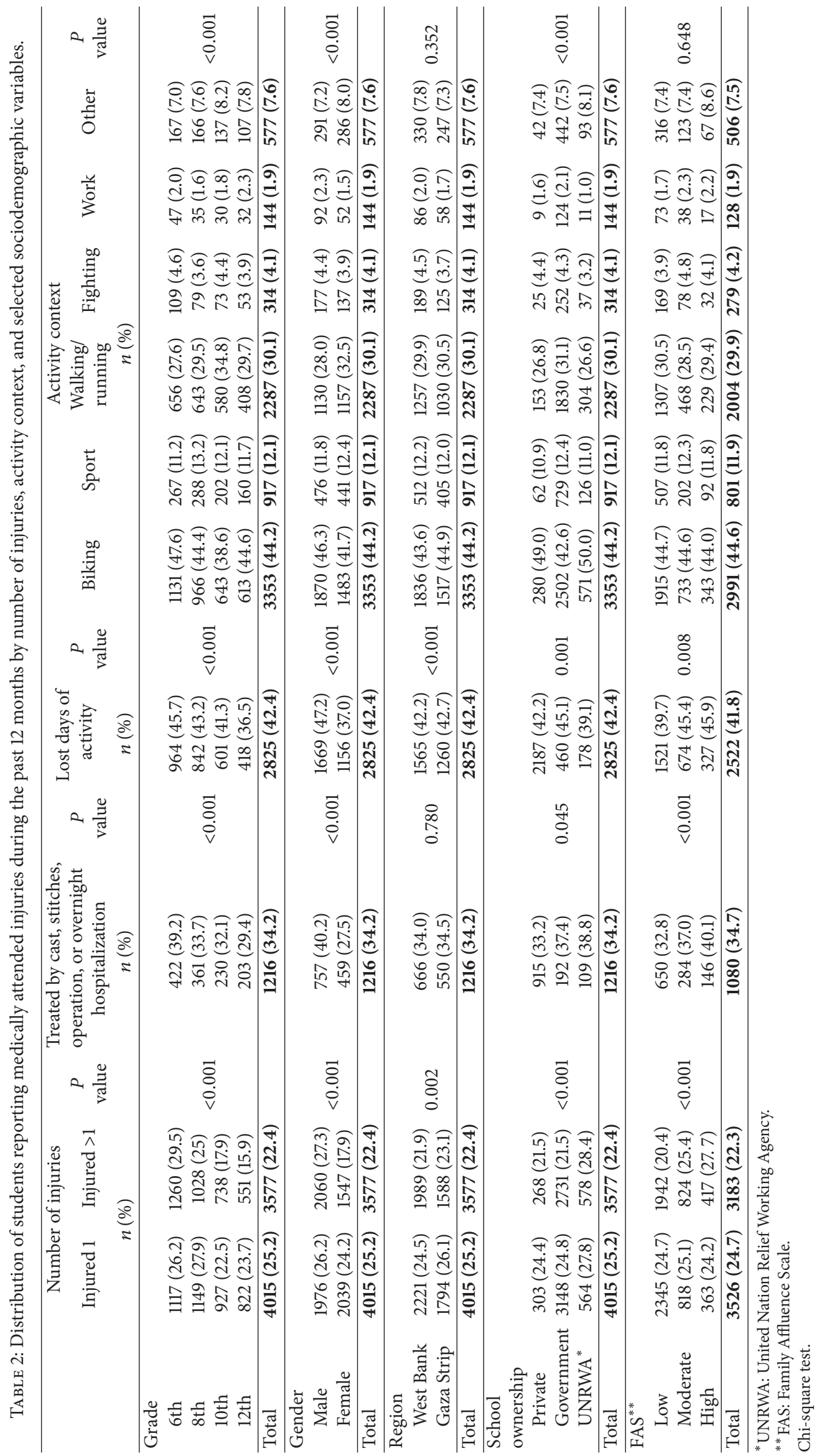


analyses that students living in low income countries reported low injury rates (e.g., Bulgaria, Romania, Slovenia) [4]. However, some researchers found contradictory results which showed that the higher the income is, the lower the death and injuries are $[34,35]$. This is the same argument used to explain the difference of injury rates among US adolescents with different SES levels [34] and undergraduate students from China [35]. As well, this study found that those living in families with higher FAS suffered from more injuries during organized activities than other students. This may be explained by the fact that richer students tend to participate more in organized activities. These results are consistent with results from another study [4].

This study shows that injury rates decreased by age: $55.7 \%$ for 6 th grade to $39.6 \%$ for 12 th grade students. These results are concurrent with most previous studies of nonfatal injuries among school adolescents [9, 10, 27]. Since Gaza Strip is a high density area with poor infrastructure, it was quite astonishing to find that injury rates were similar in both the West Bank and Gaza Strip. However, a significant difference was found $(P<0.001)$ in the probability of being injured between the different types of schools: private schools (45.9\%), government schools (46.3\%), and URWA schools (56.2\%). Most likely, this is a reflection of the composition of populations and types of neighborhoods that are served by each type of school.

In Palestine, the home was the commonest place where injuries occurred (78.5\%) to be followed by the school (9\%). Many authors in this field documented the importance of the home environment in the causation of injuries [36, 37]. And although the streets are dangerous for adolescents, this study shows that there are more hazards at home.

The vast majority of injuries among the participants were due to biking (44.2\%; boys: $46.3 \%$ and girls: $41.7 \%$ ) to be followed by running/walking (30.1\%; boys: $28.0 \%$ and girls: $32.5 \%)$. The predominance of sport injury among boys is consistent with many previous studies $[10,27,32]$. In this context, studies from Jordan and Israel reported falls as the predominant cause of injury among adolescents $[38,39]$.

This is the first study to investigate unintentional injuries in Palestine. It looked at a large population of school-aged children and is considered to be the baseline for future studies. The HBSC questionnaire items were developed continuously over several years and are supported by validation efforts $[26,40]$. The ability to examine relations between different social measures and a variety of injury types is an additional strength. Despite the strength of this study, it was a cross-sectional study, which is not strong enough to draw any causal inferences. As well, it was a self-reported school-based survey that suffers from several methodological limitations mainly: (1) recall bias, (2) "soft" measures of injury, and (3) lack of detailed etiology [29].

The problem of recall bias was discussed in depth in Harel et al., (1994) [29] showing two distinct reasons for recall bias-memory decay, resulting in loss of memory of injury events and "telescoping," resulting in inclusion of injury events that occurred outside the recall period (e.g., 13 months ago). The conclusions of Harel et al., [29] were that using a 12-month recall measure results in conservative annual estimates due to memory loss regarding minor injuries that occurred more than 3-4 months ago.

The "soft" nature of the injury measures was also discussed in previous studies [10, 28, 29]. During the past two decades, these survey items have been validated and used on many national and international surveys and have become the international standard for calculating childhood injury rates based on survey methodology $[3,8,33]$.

As a comprehensive survey, the HBSC includes a breath of topics related to the health and well-being of schoolchildren. As such, in-depth information on the exact circumstances (such as burns and poisoning) leading to individual injury events cannot be obtained. Therefore, the depth of the analyses, in terms of the etiological circumstances, is quite limited.

Injuries are a major public health issue and one that can be addressed like any other disease process that can be studied, treated, and most importantly, prevented. This study provides the first estimates of injuries among school-aged children in Palestine. It shows that injuries are common among Palestinian adolescents and despite these alarming statistics, injury remains relatively underappreciated by Palestinian health care providers, government, and the general public. We propose that Palestinian health and educational authorities should initiate the development of a national injury prevention program aimed at enhancing the safety of Palestinian school adolescents and educating the families on how to make the homes environment as safe as possible.

\section{Acknowledgments}

This survey was supported by grants from USAID MERC program, Grant no. TA-MOU-00-M20-051. The study was conducted by the Al-Quds Nutrition and Health Research Institute (ANAHRI) at Al-Quds University. Thanks for the Palestinian Ministry of Education for their assistance in the data collection procedure as well as for the Greek Scholarship Foundation (IKY) for its support to the first author C. Jildeh in her PhD study. H. Al Sabbah is a postdoctoral researcher funded by the Fulbright Scholarship, Tufts University, Boston, USA. Our gratitude goes to Mr. Manolis Linardakis for doing the data analysis.

\section{References}

[1] M. Peden, K. Oyegbite, J. Ozanne-Smith et al., "World report on child injury prevention," Tech. Rep., Unicef and WHO, 2008.

[2] M. Milton, Injuries among children and young adults in Uganda: epidemiology and prevention [Ph.D. thesis], Karolinska Institutet, Stockholm, Sweden, 2011.

[3] M. Molcho, Y. Harel, W. Pickett, P. C. Scheidt, J. Mazur, and M. D. Overpeck, "The epidemiology of non-fatal injuries among 11-, 13- and 15-year old youth in 11 countries: findings from the 1998 WHO-HBSC cross national survey," International Journal of Injury Control and Safety Promotion, vol. 13, no. 4, pp. 205211, 2006.

[4] W. Pickett, M. Molcho, K. Simpson et al., "Cross national study of injury and social determinants in adolescents," Injury Prevention, vol. 11, no. 4, pp. 213-218, 2005. 
[5] M. MacKay, A. Scanlan, L. Olsen et al., "Looking for the evidence: a systematic review of prevention strategies addressing sport and recreational injury among children and youth," Journal of Science and Medicine in Sport, vol. 7, no. 1, pp. 58-73, 2004.

[6] H. Scott, Family matters: an examination of the association between family structure and youth injury [Ph.D. thesis], University of Toronto, Toronto, Canada, 2009.

[7] W. Pickett, H. Schmid, W. F. Boyce et al., "Multiple risk behavior and injury: an international analysis of young people," Archives of Pediatrics and Adolescent Medicine, vol. 156, no. 8, pp. 786793, 2002.

[8] Y. Harel, "Injuries and youth violence," WHO: Health Behavior in School-Aged Children: Research Protocol for the 1997-1998 Survey, WHO, 1998.

[9] C. E. Currie, J. M. Williams, P. Wright, T. Beattie, and Y. Harel, "Incidence and distribution of injury among schoolchildren aged 11-15," Injury Prevention, vol. 2, no. 1, pp. 21-25, 1996.

[10] P. C. Scheidt, Y. Harel, A. C. Trumble, D. H. Jones, M. D. Overpeck, and P. E. Bijur, "The epidemiology of nonfatal injuries among US children and youth," American Journal of Public Health, vol. 85, no. 7, pp. 932-938, 1995.

[11] A. B. Bergman and F. P. Rivara, "Sweden's experience in reducing childhood injuries," Pediatrics, vol. 88, no. 1, pp. 6974, 1991.

[12] C. Onwuachi-Saunders, S. N. Forjuoh, P. West, and C. Brooks, "Child death reviews: a gold mine for injury prevention and control," Injury Prevention, vol. 5, no. 4, pp. 276-279, 1999.

[13] A. A. Hyder, M. Peden, and E. Krug, "Child health must include injury prevention," The Lancet, vol. 373, no. 9658, pp. 102-103, 2009.

[14] S. N. Bartlett, "The problem of children's injuries in low-income countries: a review," Health Policy and Planning, vol. 17, no. 1, pp. 1-13, 2002.

[15] J. A. Razzak, S. P. Luby, L. Laflamme, and H. Chotani, "Injuries among children in Karachi, Pakistan-what, where and how," Public Health, vol. 118, no. 2, pp. 114-120, 2004.

[16] W. Maziak, K. D. Ward, and S. Rastam, "Injuries in Aleppo, Syria; first population-based estimates and characterization of predominant types," BMC Public Health, vol. 6, article 63, 2006.

[17] R. A. Raanan, J. Tarairah, W. Ali et al., "Incidence of home accidents in Palestinian and Israeli communities," Research Report, 2008.

[18] Palestinian Ministry of Health- Palestinian Health Information Center (MOH-PHIC) Executive Summary, "Health status in palestine," 2005.

[19] Palestinian Central Bureau of Statistics, "Palestinian childrenissues and statistics, executive summary," 2004.

[20] Palestinian Central Bureau of Statistics, "The Population, Housing and Establishment Census 2007," in Proceedings of the Press Conference on the Preliminary Findings, (Population, Buildings, Housing Units and Establishments), Ramallah, Palestine, 2008.

[21] Palestinian Central Bureau of Statistics, "Palestine in Figures 2007," May 2008.

[22] C. E. Currie, O. Samdal, W. Boyce et al., "Health behaviour in school-aged children: a WHO cross-national study," Research Protocol For the 2001-2002 Survey, University of Edinburgh, 2002.

[23] Y. Harel and Z. Abdeen, "The Middle East Health Behavior in School-Aged Children (HBSC-ME) Cross-Cultural Study," Research Protocol for the 2004 Survey, Al Quds University and Bar Ilan University, 2003.
[24] Z. Abdeen and R. Qasrawi, "Adolescent school children in Palestine: their health and well-being," Results of the First National HBSC Survey 2004, The Al-Quds Nutrition and Health Research Institute, Al-Quds University Press, 2007.

[25] Y. Harel and Z. Abdeen, Growing Up in the Middle East: the Daily Lives and Well-Being of Israeli and Palestinian Youth, Bar Ilan University and Al Quds University, 2008.

[26] C. Currie, O. Samdal, W. Boyce et al., "Health behaviour in school-aged children: a WHO cross-national study," Research Protocol for the 2001/2002 Survey, Child and Adolescent Health Research Unit, University of Edinburgh, Edinburgh, UK, 2001.

[27] Y. Harel, Family Psychosocial Contributors to Childhood Injuries, vol. 49, no. 12, no. 8907049, Microfilm International, Ann Arbor, Mich, USA, 1988.

[28] R. J. Waxweiler, Y. Harel, and P. W. O’Carroll, "Measuring adolescent behaviors related to unintentional injuries," Public Health Reports, vol. 108, no. 1, pp. 11-14, 1993.

[29] Y. Harel, M. D. Overpeck, D. H. Jones et al., “The effects of recall on estimating annual nonfatal injury rates for children and adolescents," American Journal of Public Health, vol. 84, no. 4, pp. 599-605, 1994.

[30] W. Boyce, T. Torsheim, C. Currie, and A. Zambon, "The family affluence scale as a measure of national wealth: validation of an adolescent self-report measure," Social Indicators Research, vol. 78, no. 3, pp. 473-487, 2006.

[31] F. P. Rivara, "Epidemiology of childhood injuries. I. Review of current research and presentation of conceptual framework," American Journal of Diseases of Children, vol. 136, no. 5, pp. 399405, 1982.

[32] D. D. Lenaway, A. G. Ambler, and D. E. Beaudoin, "The epidemiology of school-related injuries: new perspectives," American Journal of Preventive Medicine, vol. 8, no. 3, pp. 193198, 1992.

[33] Y. Harel, "Rational for Injury Related Question,” in Health Behavior in School-Aged Children, A WHO Cross-National Survey: Research Protocol for the 1993-1994 Study, WHO-Regional Office for Europe, Ed., pp. 63-67, 1993.

[34] C. Cubbin, F. B. LeClere, and G. S. Smith, "Socioeconomic status and the occurrence of fatal and nonfatal injury in the United States," American Journal of Public Health, vol. 90, no. 1, pp. 7077, 2000.

[35] H. Shi, X. Yang, C. Huang, Z. Zhou, Q. Zhou, and M. Chu, "Status and risk factors of unintentional injuries among Chinese undergraduates: a cross-sectional study," BMC Public Health, vol. 11, article 531, 2011.

[36] S. P. Baker, "Prevention of childhood injuries," Medical Journal of Australia, vol. 1, pp. 466-470, 1980.

[37] M. H. Hall, "Hazards to children in the home environment," Community Health, vol. 5, no. 5, pp. 238-245, 1974.

[38] S. Janson, M. Aleco, A. Beetar, A. Bodin, and S. Shami, "Accident risks for suburban preschool Jordanian children," Journal of Tropical Pediatrics, vol. 40, no. 2, pp. 88-93, 1994.

[39] A. Broides and M. Assaf, "Home accidents in Arab Bedouin children in southern Israel," Journal of Child Health Care, vol. 7, no. 3, pp. 207-214, 2003.

[40] C. E. Currie, Ed., "Health Behaviour in School-aged Children: research protocol for the 1997-1998 survey," WHO Coordinating Center for the Study of Health Behaviour in School-Aged Children, Edinburgh, UK, 1998. 

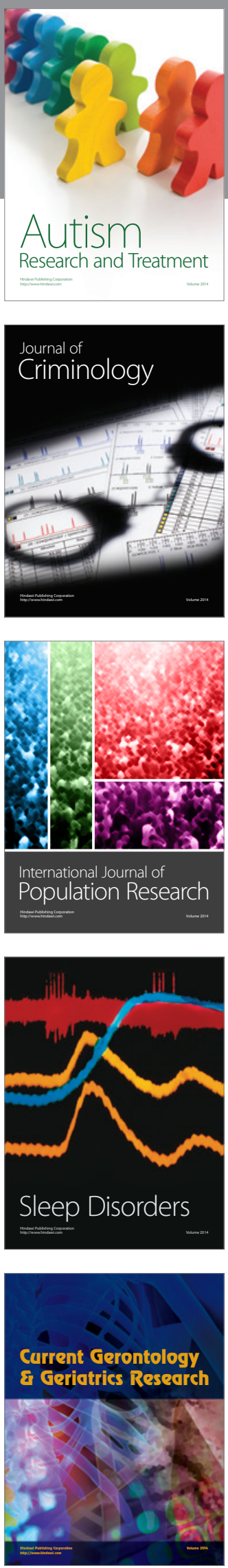
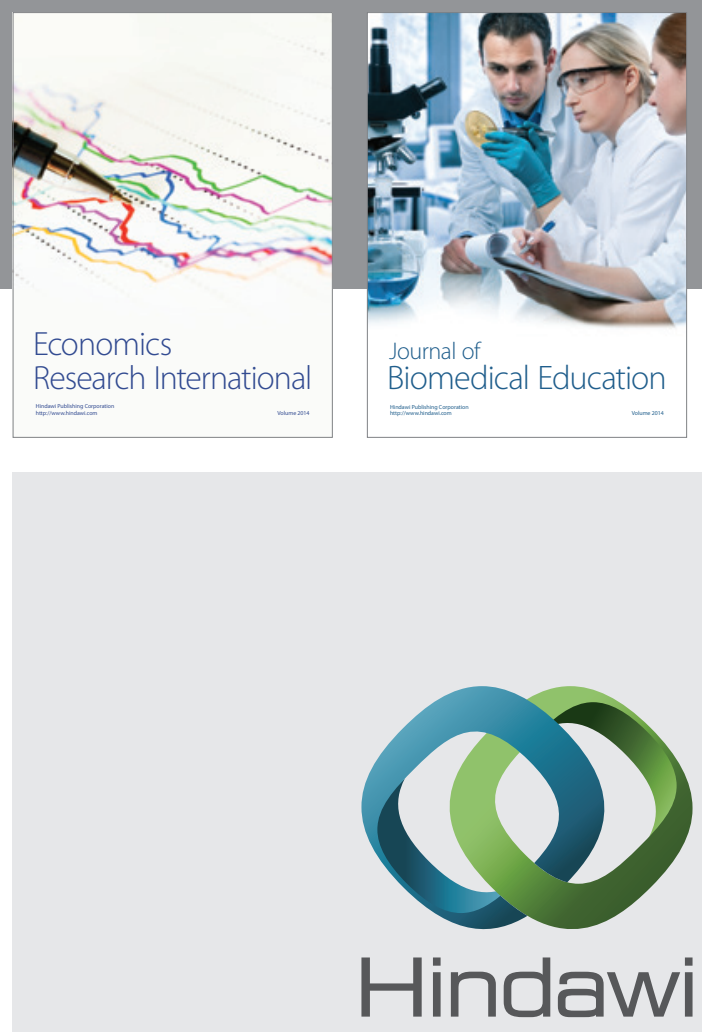

Submit your manuscripts at

http://www.hindawi.com
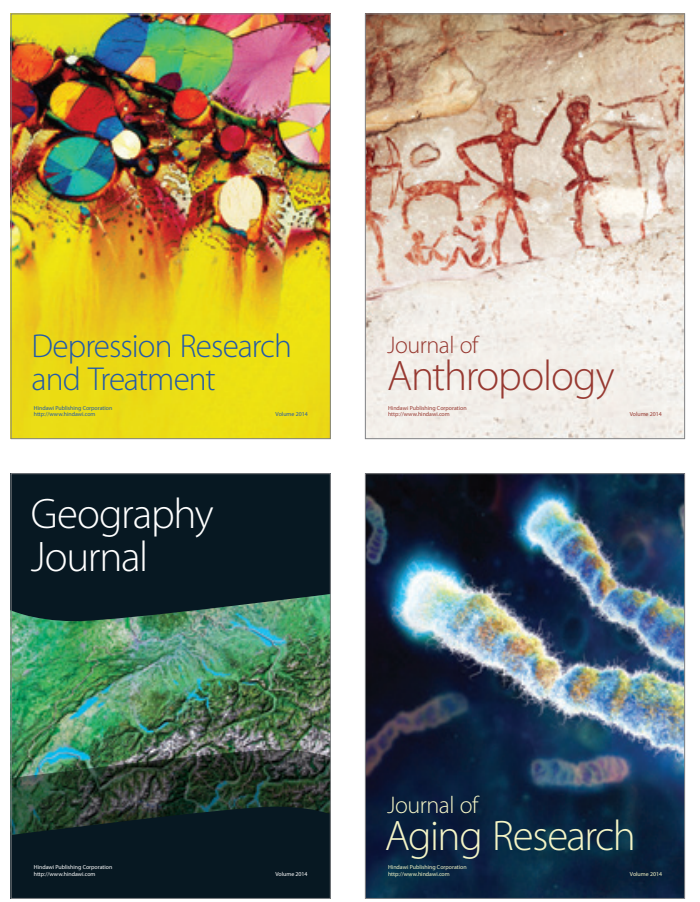
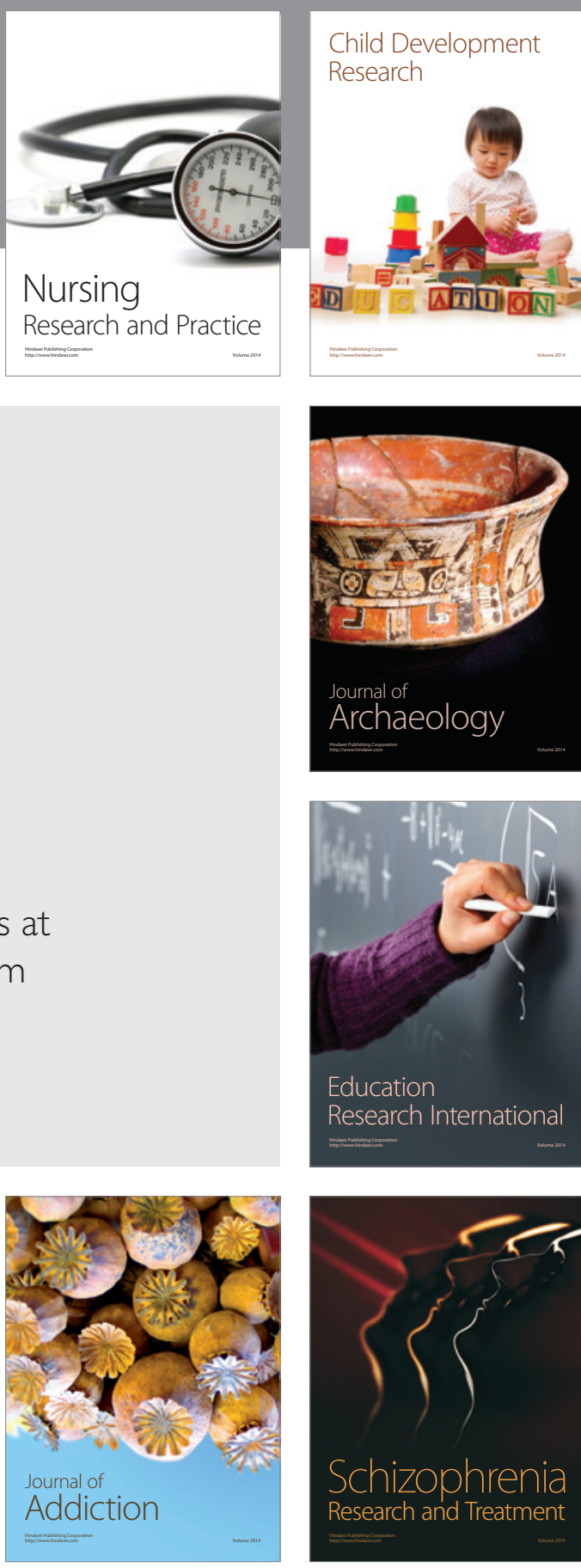

(D)
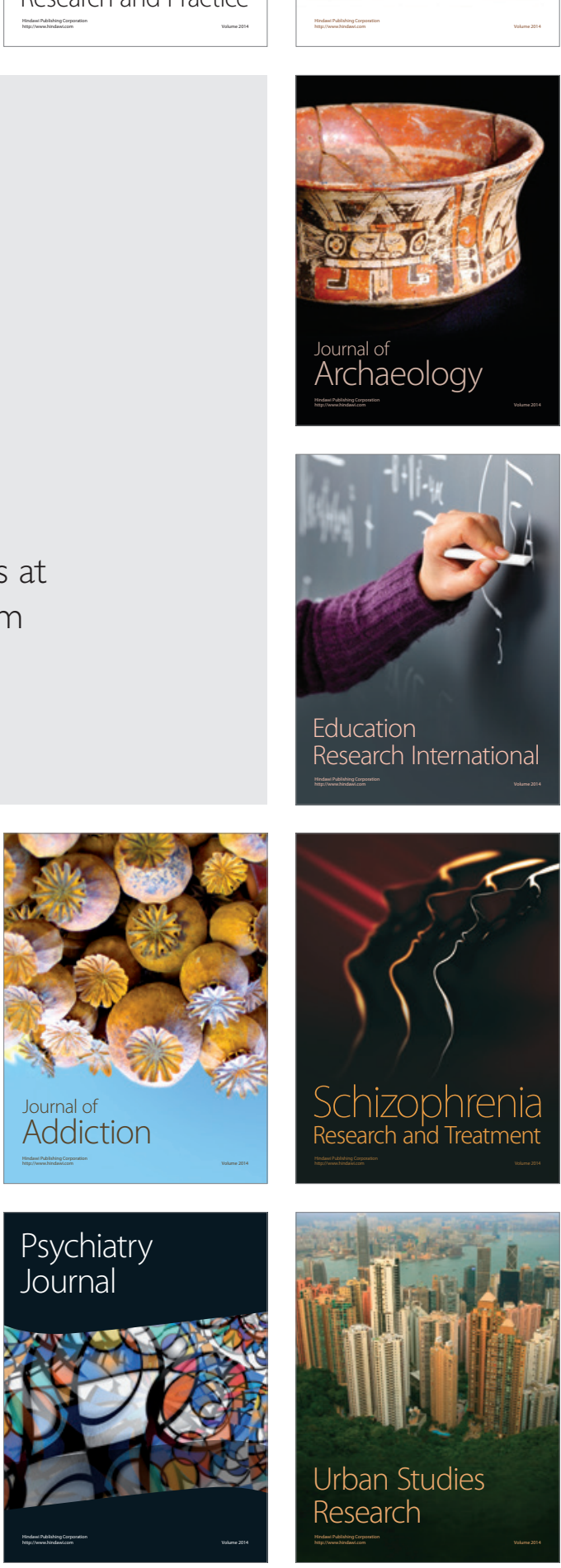